\title{
ARCHEI \\ ARCH ENINE \\ PRONTUÁRIO ONCOLÓGICO COMO FONTE DE INFORMAÇÃO NA ASSISTÊNCIA AO PACIENTE COM CÂNCER
}

\author{
Amanda Damasceno de Souza ${ }^{1}$ \\ Marília de Abreu Martins de Paiva ${ }^{2}$
}

\section{RESUMO}

Aborda o arquivo de prontuários do setor de Oncologia sob a perspectiva de fonte de informação para assistência e vigilância do câncer. Como objetivo a pesquisa analisou o Prontuário do Paciente da Oncologia na coleta de informação sobre câncer. Trata-se de pesquisa de base documental, a partir de documentos regulatórios (legislativos e infra legislativos) sobre a informação à saúde em geral, e ao documento prontuário do paciente, em particular. Os resultados da pesquisa apontaram que as mudanças ocorridas no prontuário, ao longo do tempo, foram relacionadas à melhora no detalhamento das informações sobre o paciente, adição de novos exames conforme ocorreu evolução no tratamento do câncer e criação de legislação para atender as demandas da versão eletrônica. Descreve as questões sobre obrigatoriedade, sigilo, guarda, acesso, guarda e temporalidade. No prontuário da oncologia, informações epidemiológicas foram acrescidas devido ao Registro Hospitalar de Câncer. Concluímos que a utilização do prontuário vai além da assistência ao paciente e atividades administrativas, sendo este utilizado para estudos epidemiológicos, pesquisa clínica, trabalhos científicos e acadêmicos, evolução da doença, dos tratamentos realizados e para coleta de informações em oncologia para as estimativas de câncer no Brasil do Instituto Nacional do Câncer.

Palavras-Chave: Documentos arquivísticos. Arquivos médicos. Prontuário do paciente. Prontuário Eletrônico do Paciente. Registro Hospitalar. Neoplasias.

\section{ONCOLOGY MEDICAL RECORD AS A SOURCE OF INFORMATION IN THE CARE FOR PATIENTS WITH CANCER}

\section{ABSTRACT}

It addresses the use of medical records of the Oncology field as a possible source of information for cancer care and surveillance. As a goal, the research investigated Oncology Patient Records in the collection of information about cancer. This is a document-based research, based on regulatory documents (legislative and infra-legislative) about general health information and on patient's charts, in particular. The results of the research showed that the changes that occurred in the medical chart over time were related to improvements in the detailing of information about the patient, the addition of new tests as cancer treatments advanced, and the creation of a legislation to meet the requirements of electronic patient records. Epidemiological information was added due to a new Cancer Registry. It was concluded that the use of medical records goes beyond patient care and administrative activities, being also used for epidemiological studies, clinical trials,

${ }_{1}^{1}$ Doutoranda em Gestão e Organização do Conhecimento / UFMG / amanda@ufmg.br

2 Doutora em Ciência da Informação / UFMG / biblio.marilia@gmail.com 
clinical research, scientific and academic work, as well as disease evolution, and collection of oncology data for the Brazilian National Cancer Institute

Keywords: Archival documents. Medical archives. Medical records. Electronic Health Record. Cancer Registries. Neoplasms.

\section{INTRODUÇÃO}

Cada vez se reconhece o valor dos documentos arquivísticos para as diversas áreas do conhecimento humano. Rosseau e Couture, em seu vasto "Os fundamentos da disciplina arquivística" descrevem a história e a função dos arquivos através das civilizações e atribui ao arquivista do final do séc. XX o papel de "gestor de informação" assumindo que o documento arquivístico, com seu triplo papel "cultural, administrativo e científico", passa a impor novas demandas sociais. (ROSSEAU; COUTURE, 1998, p.4647).

No presente trabalho, abordamos uma tipologia específica de documento da área de saúde, o prontuário do paciente, em seu contexto administrativo Serviço de Arquivo Médico e Estatístico (SAME). 0 prontuário do paciente, assim considerado fonte de informação dentro da unidade de informação SAME tem enorme potencialidade informativa para a pesquisa médica, devendo garantir uma segura e específica comunicação entre membros da equipe multidisciplinar em saúde, para fins de auxílio ao diagnóstico e acompanhamento e tomada de decisão jurídica e administrativa.

A espécie documental prontuário, segundo Belloto é um documento "informativo", ou seja, "são opinativos/enunciativos e esclarecem questões contidas em outros documentos, cujo conteúdo vai fundamentar uma resolução: pareceres, informações, relatórios (...)"e "acompanham o desempenho dos interessados na sua atuação profissional em (...) tratamentos médicos e psicológicos. (..)." (BELLOTO, 2002, p. 27-77). Além disso, por estar aplicado em especialidade médica, no nosso caso, constitui-se um tipo documental especializado que servirá a funções específicas que lhes derem o corpo médico envolvido. Contudo, o prontuário também pode ter valor (interesse e utilidade) secundário, assim sua preservação também deverá levar em conta objetivos como, ser fonte de memória institucional, fonte de informação secundária em saúde, sobre doença ou período específico, para a pesquisa clínica e para publicações científicas e epidemiológicas. Assim, o prontuário médico apresentará valores secundários que irão transcender o propósito imediato para qual foi produzido, 
sendo necessária sua preservação a longo prazo. (GREENE, 2008).

Os documentos de arquivo, pela perspectiva de seu ciclo de vida, têm três fases ou idades, em que se denominam arquivos correntes, intermediários e permanentes. Durante sua vida, os documentos suprem diferentes funções e interesses dentro e fora de sua instituição criadora, e tem seus valores modificados, neste contexto. Ainda conforme Rosseau e Couture, ao descreverem esse ciclo de vida, as etapas não são tão "distintas e sem ligação." (ROSSEAU; COUTURE, 1998, p.117). Muito importante para a compreensão da vida do documento são os seus valores: primário ou secundário. 0 valor primário dos documentos arquivísticos é a "qualidade de um documento baseado nas utilizações imediatas e administrativas que lhe deram os seus criadores." Enquanto seu valor secundário é "a qualidade de um documento baseada nas utilizações não imediatas, ou científicas. Esta qualidade radica essencialmente no testemunho privilegiado e científico que o documento fornece." (ROSSEAU, COUTURE, 1998, p.117118).

O ciclo de vida de um documento é determinado pelo instrumento arquivístico denominado Tabela de Temporalidade e Destinação de Documentos (TTDD), que estabelece os prazos de permanência do documento em cada fase: corrente, intermediária e sua destinação final de eliminação ou recolhimento ao arquivo permanente. A valoração baseada no interesse e uso do documento irá determinar o tempo necessário de sua permanência no arquivo em cada fase. Em relação ao prontuário do paciente, sua temporalidade é regulamentada pelo Conselho Federal de Medicina (CFM) na Resolução no 1.821 de 2007. (CONSELHO FEDERAL DE MEDICINA, 2007). 0 cumprimento do ciclo de vida dos documentos por meio da aplicação da tabela de temporalidade é de fundamental importância para a gestão de documentos dentro da instituição.

A construção da revisão da literatura desta pesquisa abrangeu a legislação vigente do prontuário do paciente em papel e meio eletrônico. Para isso foram descritas as principais características deste documento, forma de organização, aspectos éticos de obrigatoriedade, sigilo, guarda e sua importância como fonte de informação no tratamento do câncer na coleta de dados para o Registro Hospitalar de Câncer (RHC). 0 objetivo da pesquisa foi analisar o Prontuário do Paciente do setor de Oncologia como fonte para a coleta de informação sobre câncer. A partir dessa análise, pretende-se demonstrar os valores primários e secundários dessa tipologia documental, que justifica 
um tempo de guarda maior.

\subsection{METODOLOGIA}

Trata-se de pesquisa de base documental, a partir de documentos regulatórios (legislativos e infra legislativos) sobre a informação à saúde em geral, e ao documento prontuário do paciente, em particular. Para melhor exemplificar as necessidades informacionais e o uso dos documentos, fez-se o recorte específico para pacientes de oncologia de um arquivo de hospital público de Belo Horizonte. A abordagem foi exploratória, do ponto de vista dos procedimentos técnicos, envolveu levantamento bibliográfico e análise documental. (GIL, 1994).

\section{PRONTUÁRIO DO PACIENTE}

Ao abordarmos o assunto prontuário, percebemos variedade de termos: prontuário médico, prontuário nosológico do paciente, prontuário médico do paciente e também termos relacionados à sua documentação: laudo médico, relatório médico, exames médicos. (CRMDF, 2006). Entretanto, no presente estudo adotaremos a expressão Prontuário do Paciente que segundo o Conselho Regional de Medicina do Distrito Federal (CRMDF), é o termo politicamente mais adequado. Teixeira (2007) ressalta que são diversos os profissionais da saúde que atuam no atendimento do paciente: médicos, pessoal de enfermagem, assistentes sociais, psicólogos, terapeutas ocupacionais, nutricionistas, farmacêuticos, dentre outros. Uma vez que o prontuário não recebe somente anotações de médicos, sendo que dados de saúde de pacientes e populações são coletados por uma variedade de profissionais de saúde, a denominação mais indicada é prontuário do paciente e não prontuário médico. (SHORTLIFFE; BARNETT, 2014; CRMDF, 2006).

Do ponto de vista legal, a elaboração do prontuário do paciente foi definida como obrigatória no artigo n. 69 Código de Ética Médico (CFM, 2010). Regulamentado pela Resolução do Conselho Federal de Medicina (CFM, 1988) n.1.246, de 1988, e revogada pela resolução n. 1.931, de 2009 (CFM, 2009). Entretanto, o conceito de prontuário é apresentado na resolução n.1.638, de 2002, que assim o define, artigo1ํ: 
documento único constituído de um conjunto de informações, sinais e imagens registradas, geradas a partir de fatos, acontecimentos e situações sobre a saúde do paciente e a assistência a ele prestada, de caráter legal, sigiloso e científico, que possibilita a comunicação entre membros da equipe multiprofissional e a continuidade da assistência prestada ao indivíduo. (CFM, 2002).

A resolução n.1821, de 2007, considera que o médico tem o dever de elaborar um prontuário para cada paciente a que assiste, e as unidades de serviços de apoio, diagnóstico e terapêutica têm documentos próprios, que fazem parte dos prontuários dos pacientes. (CFM, 2007).

As práticas de notificação em Oncologia começaram em 1975 influenciados pela da criação do Programa de Controle ao Câncer (PCC), desenvolvido através de um convênio firmado entre o Ministério da Previdência e Assistência Social (MPAS) e o Ministério da Saúde, com objetivo de universalização dos procedimentos relacionados à doença e com vistas a integrar as ações de controle do câncer. (BARRET0, 2005). Desde então, o prontuário do paciente oncológico apresenta as seguintes funções:
a) Assistência ao paciente;
b) Ganho de tempo;
c) Apoio diagnóstico;
d) Estatística;
e) Cobrança;
f) Defesa;
g) Pesquisas científicas e ensino;
h) Informações epidemiológicas;
i) Eficiência dos profissionais;
j) Meios de Comunicação;
k) Elaboração de relatórios e atestados.

O prontuário é importante para o paciente por possibilitar uma melhor assistência no diagnóstico, atendimento e tratamento da doença (CRMDF, 2006). 


\subsection{CARACTERÍSTICAS DO PRONTUÁRIO}

Os registros documentais apresentam características relacionadas à função que exercem, essas características são citadas por Duranti (1994, p. 2-4):

Imparcialidade ${ }^{3}$ - os registros são inerentemente verdadeiros, as razões por que eles são produzidos (para desenvolver atividades) e as circunstâncias de sua criação (rotinas processuais) asseguram que não são escritos na intenção ou para informação da posteridade, nem com a expectativa de serem expostos ou com receio do olhar público. Os documentos trazem uma promessa de fidelidade aos fatos e atos que alguns interesses não gostariam de ver revelados.

Autenticidade - os documentos são autênticos porque são criados tendose em mente a necessidade de agir através deles, são mantidos com garantias para futuras ações ou para informações... Assim, os documentos são autênticos porque são criados, mantidos e conservados sob custódia de acordo com procedimentos regulares que podem ser comprovados.

Naturalidade - diz respeito a maneira como são acumulados no curso das transações, não são coletados como objetos de coleções, mas acumulados naturalmente nos escritórios em função dos objetos práticos da administração. Acumulam-se de maneira continua e progressiva.

Inter-relacionamento - os documentos estabelecem relações no decorrer do andamento das transações e de acordo com suas necessidades. Os documentos estão ligados entre si por um elo que é criado no momento em que são produzidos ou recebidos, que é determinado pela razão de sua produção e que é necessário à sua própria existência, à sua capacidade de cumprir seus objetivos, ao seu significado, confiabilidade e autenticidade.

Unicidade - a unicidade provém do fato de que cada registro documental assume um lugar único na estrutura documental do grupo ao qual pertence e no universo documental.

Duranti (1994) afirma que pelas características dos registros documentais de imparcialidade, autenticidade, naturalidade, inter-relacionamento e unicidade, podemos compreender nosso passado imediato ou histórico, com propósitos administrativos e culturais.

Em relação as característica do prontuário como registro documental, essas são regulamentadas por resoluções do CFM que considera o prontuário como um :

\footnotetext{
${ }^{3}$ Grifo da autora Duranti.
} 
documento valioso para o paciente, para o médico que o assiste e para as instituições de saúde, bem como para o ensino, a pesquisa e os serviços públicos de saúde, além de instrumento de defesa legal. (CFM, 2002).

O CFM regulamenta a necessidade de guarda, qualidade, preservação das informações, armazenamento, a eliminação de documentos do prontuário obedecendo aos critérios médico-científicos, históricos e sociais de relevância para o ensino, a pesquisa e a prática médica, a criação da comissão de revisão de prontuário. Entretanto, confere a legislação arquivística brasileira através do Conselho Nacional de Arquivos (CONARQ), a normatização em relação a temporalidade e a classificação dos prontuários na resolução n. 1.821, de 2007. (CFM, 2007; BARROWS; CLAYTON, 1996).

\subsection{OBRIGATORIEDADE (NATURALIDADE)}

Segundo o Código de Ética Médica publicado pelo CFM na resolução n. 1931, de 17 de setembro 2009, no capítulo X sobre documentos médicos, regula sobre a obrigatoriedade do médico em elaborar o prontuário e sobre a responsabilidade de guarda "É vedado ao médico: Art. 87. Deixar de elaborar prontuário legível para cada paciente". A resolução descreve os dados clínicos necessários ao prontuário, e relata que o mesmo deve ser preenchido, em cada avaliação. A ordem dos dados é cronológica contendo também a data, hora, assinatura e número de registro do médico no Conselho Regional de Medicina. (CFM, 2010).

\subsection{SIGILO (ACESSO CONTROLADO)}

A privacidade de informações coletadas durante o cuidado médico dos pacientes oncológicos é necessária por causa do significado econômico, psicológico e os danos sociais que pode causar ao indivíduo quando suas informações pessoais de saúde são divulgadas. Por isso, o sigilo médico é previsto em lei de acordo com o CONARQ (2005). Sobre o sigilo médico, a resolução n.1821 de 2007, regulamentada pelo CFM, prevê que: 
CONSIDERANDO que toda informação em saúde identificada individualmente necessita de proteção em sua confidencialidade, por ser principio basilar do exercício da medicina;

CONSIDERANDO os enunciados constantes nos artigos 102 a 109 do Capítulo IX do Código de Ética Médica, o médico tem a obrigação ética de proteger o sigilo profissional. (CFM, 2007).

Segundo o Código de Ética Médica publicado pelo CFM em sua resolução n.1931, de 17 de setembro 2009, no capítulo IX, página 61, sobre sigilo profissional, prevê que:

É vedado ao médico:

Art. 73. Revelar fato de que tenha conhecimento em virtude do exercício de sua profissão, salvo por motivo justo, dever legal ou consentimento, por escrito, do paciente.

Parágrafo único. Permanece essa proibição: a) mesmo que o fato seja de conhecimento público ou o paciente tenha falecido; b) quando de seu depoimento como testemunha. Nessa hipótese, o médico comparecerá perante a autoridade e declarará seu impedimento; c) na investigação de suspeita de crime, o médico estará impedido de revelar segredo que possa expor o paciente a processo penal. (CFM, 2007).

Em relação ao sigilo profissional, o médico deverá respeitar a privacidade e não poderá relatar em publicações de casos clínicos, informações que possam identificar o paciente ou divulgar em meios de comunicação, imagens de pacientes. Em exames médicos de trabalhadores não é permitido revelar informações confidenciais ou fornecer para empresas seguradoras sem o consentimento do representante legal do paciente, as causas da morte do paciente sob seus cuidados, além das informações descritas na declaração de óbito. (CFM, 2010). A resolução também aborda as orientações à equipe auxiliar médica em respeitar o sigilo profissional.

\subsection{ACESSO, GUARDA E TEMPORALIDADE}

Em relação ao acesso, o Código de Ética Médica publicado pelo CFM prevê é permitido o manuseio e o conhecimento dos prontuários por pessoas não obrigadas ao sigilo profissional quando sob a responsabilidade do médico (CFM, 2010).

O Código de Ética Médica relata que ao paciente, é permitido o acesso a seu prontuário, podendo o mesmo solicitar cópia e explicações necessárias à sua compreensão. Desde que não ocasionem riscos ao próprio paciente ou a terceiros. Para a liberação de cópias do prontuário é necessária a autorização por escrito do paciente. Em 
caso de requisição judicial, o prontuário ou sua cópia poderá ser disponibilizado ao perito médico, nomeado pelo juiz (CFM, 2010).

A responsabilidade de guarda do prontuário em papel é do médico ou da instituição que assiste o paciente (CFM, 2010-). O CFM, regulamentado na resolução n. 1821 de 2007 artigo 3ํㅡㄹ dispõe sobre a guarda em formatos eletrônicos. A resolução 3ำ autoriza o uso de sistemas informatizados para a guarda e manuseio de prontuários de pacientes e também para a troca de informação identificada em saúde. 0 registro em papel poderá ser eliminado desde que os sistemas informatizados atendam integralmente aos requisitos dos níveis: Nível de Garantia de Segurança 1 (NGS1) e Nível de Garantia de Segurança 2 (NGS2). Os requisitos são descritos no Manual de Certificação para Sistemas de Registro Eletrônico em Saúde (http://www.sbis.org.br/certificacao/Manual_Certificacao_SBIS-CFM_2016_v4-2.pdf) (CFM, 2007; SILVA; VIRGINIO JUNIOR, 2016).

O CFM (2007) dispõe também sobre a eliminação e guarda permanente nos artigos:
Art. 6ำ No caso de microfilmagem, os prontuários microfilmados poderão ser eliminados de acordo com a legislação específica que regulamenta essa área e após análise obrigatória da Comissão de Revisão de Prontuários da unidade médico-hospitalar geradora do arquivo.
Art. 7ํㅡ Estabelecer a guarda permanente, considerando a evolução tecnológica, para os prontuários dos pacientes arquivados eletronicamente em meio óptico, microfilmado ou digitalizado.
Art. 8o Estabelecer o prazo mínimo de 20 (vinte) anos, a partir do último registro, para a preservação dos prontuários dos pacientes em suporte de papel, que não foram arquivados eletronicamente em meio óptico, microfilmado ou digitalizado.
Art. 9o As atribuições da Comissão Permanente de Avaliação de Documentos em todas as unidades que prestam assistência médica e são detentoras de arquivos de prontuários de pacientes, tomando como base as atribuições estabelecidas na legislação arquivística brasileira, podem ser exercidas pela Comissão de Revisão de Prontuários. (CFM, 2007).

Em relação a guarda em formatos eletrônicos, a resolução n.1821 de 2007 teve seu artigo $10^{\circ}$ sobre a expedição do selo de qualidade dos sistemas informatizados, revogado pela Resolução CFM n. 2.218, de 24 de outubro de 2018. Em relação a guarda do prontuário em papel ou sua cópia digitalizada, cabe a instituição, após cumprir o tempo estabelecido pela resolução, prorrogar esse prazo de guarda, para atender as finalidades do prontuário como fonte de pesquisa científica, clínica e epidemiológica. 
A seguir apresentaremos as características do prontuário do paciente na versão eletrônica desse documento, conhecida como Prontuário Eletrônico do Paciente (PEP).

\section{PRONTUÁRIO ELETRÔNICO DO PACIENTE (PEP)}

A implementação de Prontuário Eletrônico do Paciente (PEP) representa um desafio para instituições de saúde no Brasil e para a assistência médica. Isso devido ao suporte de informática necessário para sua realização e aos padrões de norma e certificações estabelecida pelo Conselho Federal de Medicina (CFM) e pela Sociedade Brasileira de Informática em Saúde (SBIS) em relação aos critérios de segurança da informação (BEZERRA, 2009), e do certificado digital emitido no âmbito da Infraestrutura de Chaves Públicas Brasileiras (ICP-Brasil). (COSTA, 2012). Embora tenhamos um expressivo avanço das tecnologias de saúde nos últimos 20 anos, os prontuários em papel ainda são amplamente utilizados na assistência ao paciente. (GALLIGIONI et al., 2009).

Segundo o Institute of Medicine ${ }^{4}$ o Prontuário Eletrônico do Paciente é:

um registro eletrônico que reside em um sistema especificamente projetado para apoiar os usuários fornecendo acesso a um completo conjunto de dados corretos, alertas, sistemas de apoio à decisão e outros recursos, como links para bases de conhecimento médico. (IOM, apud MASSAD; MARIN; AZEVEDO NETO, 2003, p.6).

Entretanto, Massad, Marin e Azevedo Neto (2003), relatam algumas desvantagens do PEP em relação ao prontuário em papel como:

a) Necessidade constante de treinamento e grande investimento de hardware e software;

b) Apresentar dificuldades aos usuários no seu manuseio, acarretando assim resistências a sua utilização e até mesmo sabotagens;

c) Demora em obter os resultados do investimento;

d) O PEP está sujeito às falhas de software e hardware. A falta de energia impossibilita sua utilização.

4 INSTITUTE OF MEDICINE (IOM). The computer-based patient record: an essencial technology for heath care. revised edition. Washington (D.C): Division of Health Care Services, Institute of Medicine, National Academy of Science, 1997. 
Na implantação do PEP é necessária a cooperação da equipe multidisciplinar, e sua educação na implantação de normas e padrões tecnológicos e em relação aos dados. Bezerra (2009) relata que o PEP trará muitas contribuições tanto para equipe médica como as Unidades Hospitalares e pacientes, uma vez que proporcionará rapidez, qualidade da informação clínica, confiabilidade dos dados que poderão ser usados na gerência, assistência direta ao paciente, nas pesquisas clínicas, nas auditorias, além de permitir a criação de indicadores administrativos, médicos assistenciais, gerenciais nos processos de avaliação da qualidade hospitalar.

Uma vez que o câncer é uma doença complexa e pode exigir múltiplas estratégias terapêuticas, essa complexidade pode se beneficiar da tecnologia e assim melhorar o tempo de resposta e a precisão da informação, bem como a qualidade do tratamento do câncer. (GALLIGIONI et al., 2009).

As questões principais que norteiam o PEP são em relação a segurança e sigilo das informações dos pacientes. Sua utilização deve obedecer técnicas concernentes à digitalização e uso dos sistemas informatizados para a guarda e manuseio dos documentos dos prontuários dos pacientes, autorizando a eliminação do papel e a troca de informação identificada em saúde do CFM regulamentado pela resolução n. 1.821 de 2007. (CFM, 2017).

O governo federal criou lei n. 13.787 de 27 de dezembro de 2018, que dispõe sobre a digitalização e a utilização de sistemas informatizados para a guarda, o armazenamento e o manuseio de prontuário de paciente. Sobre a preservação dos documentos de valor histórico, compete a legislação arquivística definir sobre sua preservação. A lei permite a eliminação dos prontuários em papel ou digitalizados, após a vigência mínimo de 20 (vinte) anos a partir do último registro. Permite também a devolução do prontuário ao paciente. (BRASIL, 2018).

Independente do prontuário ser em papel ou em formato eletrônico, esse é fonte de informação da mais alta relevância na assistência ao paciente com câncer.

\section{O PRONTUÁRIO COMO FONTE DE INFORMAÇÃO NO TRATAMENTO DO CÂNCER}

Para uma melhor assistência ao paciente é necessário que suas informações estejam organizadas e arquivadas adequadamente para utilização pelo médico e equipe 
de saúde. Nesse contexto, o prontuário do paciente se configura como um documento de extrema importância na atenção do paciente em hospitais gerais e com serviço de oncologia em clínicas especializadas em oncologia, no atendimento ao paciente com câncer. No âmbito da Oncologia, o prontuário cumpre a obrigatoriedade de ter organizados os documentos pessoais, resultados de exames, história clínica e evolução da doença, dos pacientes tratados no hospital, para fins de assistência.

Prontuário do paciente é um conjunto de documentos padronizados, ordenados e concisos, destinados ao registro dos cuidados médicos, paramédicos e multidisciplinares prestados ao paciente pelos estabelecimentos de saúde. (TEIXEIRA, 2007). 0 prontuário do paciente é imprescindível como dispositivo de atendimento médico-hospitalar e se constitui meio de prova judicial. É constituído por um conjunto de informações, sinais e imagens registradas, geradas a partir de fatos, acontecimentos e situações sobre a saúde do paciente e a assistência que foi prestada a ele. Apresenta caráter legal, sigiloso e científico e ainda possibilita a comunicação entre membros da equipe multidisciplinar para dar continuidade à assistência prestada ao paciente. (TEIXEIRA, 2007).

Ao abordarmos a busca de informação em prontuários, possui um valor arquivístico primário de informar acerca da trajetória clínica, mas também por servir de prova jurídica e administrativa. Característica que requer acurácia nas anotações realizadas. 0 prontuário é fonte de informação que permite avaliar a qualidade do tratamento prestado ao paciente em instituições de saúde. Vai além, ao apresentar um potencial valor arquivístico secundário por disponibilizar dados e informações para realização de pesquisa científica e clínica. No âmbito da Oncologia, enfoque deste estudo, o prontuário também é fonte de informação para a realização de pesquisa científica e clínica em oncologia (KANAS et al., 2010), para a vigilância e as estimativas de câncer no Brasil. (INCA, 2018).

O prontuário do paciente não se encontra categorizado em uma tipologia de fonte de informação na literatura, seja como fontes primarias, secundarias ou terciárias. Os documentos de fontes primárias, em sua produção há interferência do autor, por exemplo, relatórios técnicos, trabalhos apresentados em congressos, teses, dissertações, patentes e artigo científicos. As fontes secundárias, são caracterizadas por facilitar o uso do conhecimento, por exemplos as enciclopédias, manuais, dicionários, anuários, entre outras. Por fim, as fontes terciárias, guiam os usuários para utilizar as fontes primárias e 
secundárias, são exemplos às bibliografias, catálogos, diretórios, serviços de indexação, etc. (MUELLER, 2000). Entretanto, devido ao seu formato de apresentar dados e informações coletadas diretamente da fonte, ou seja, do paciente, e pela interferência do autor na sua produção, o prontuário poderia ser considerado uma fonte de informação primária. Isso porque a partir dos dados registrados no cadastro do paciente, na anamnese, na evolução médica, nos exames laboratoriais, nos laudos, entre outros, é que serão realizados pesquisas clínicas e trabalhos científicos que darão origem a monografias, dissertações, teses e artigos na área de saúde.

Para o cuidado ao paciente com câncer é necessário o registro de seus dados básicos como: nome, endereço, filiação, estado civil, procedência, naturalidade. Seus dados epidemiológicos: raça se possui histórico familiar de câncer, se fez uso de álcool e tabaco. Seus dados biométricos: peso e altura. Além de documentos de identificação: carteira de identidade e Comprovante de Situação Cadastral (CPF). 0 registro dessas informações é necessário para conhecer melhor o paciente.

Após essa primeira etapa, o médico irá realizar a consulta e elaborar a anamnese (documento que irá relatar a história do paciente e os motivos que o levaram a procurar o serviço de saúde). Na terceira etapa, o médico irá solicitar exames para descobrir qual é o tipo de câncer. Nesse momento são solicitados exames de punção, como biópsia; e exames de imagem, como tomografia, raio-X. Todos esses procedimentos resultam na criação de documentos que necessitam ser organizados, para então, o médico definir o tratamento do paciente. A organização desses documentos origina o prontuário do paciente.

A organização de documentos do paciente segue uma ordem cronológica e contém os seguintes informações e/ou documentos (BERTOLLI FILHO,1996, p.175):
a) Cadastro do paciente;
b) Termo de consentimento livre e esclarecido;
c) Folha de anamnese, exame físico e dados etiológicos;
d) Evolução e prescrição médica;
e) Exames complementares (laboratoriais, radiológicos, ultra-sonográficos e outros) e seus respectivos resultados; resumo de alta; registro da informação de óbito;
f) Cópia de Documentos de identificação. 
O prontuário do paciente da Oncologia além de ser fonte de informação para pesquisas é fonte para dados estatísticos de incidência, prevalência e mortalidade, na vigilância do câncer. Com esses dados, as ações de vigilância visam conhecer, detectar e prevenir fatores que determinam o processo saúde e doença, auxiliar a elaboração de políticas públicas e serviços que garantam o direito à saúde das pessoas com câncer. (INCA, 2018). As estatísticas de câncer no Brasil são elaboradas pelo Instituto Nacional do Câncer (INCA), por meio de coleta de dados nos prontuários realizada pelo Registro Hospitalar de Câncer (RHC).

\subsection{O REGISTRO HOSPITALAR DE CÂNCER (RHC)}

Os Registros Hospitalares coletam somente dados de pacientes atendidos no hospital, com diagnóstico confirmado de câncer. (INCA, 2010). Os registros de câncer são sistemas de coleta, armazenamento, processamento e análise de dados, por localização primária do tumor e comportamento dessa patologia, em uma área geográfica específica ou unidade hospitalar. Os registros podem ser basicamente diferenciados segundo INCA (2010a, b):

a) Registro de Câncer de Base Populacional (RCBP): coletam informações sobre incidência, tendências e sobrevida.

b) Registro Hospitalar de Câncer (RHC): coletam dados referentes ao diagnóstico, tratamento e evolução dos casos de neoplasia maligna.

Enquanto os RCBP produzem informações com a finalidade de descrever e monitorar o perfil da incidência de câncer no país, os RHC reúnem informações para avaliação da qualidade da assistência prestada a pacientes atendidos em determinado hospital ou num conjunto de hospitais. (INCA, 2010a).

O Registro Hospitalar de Câncer (RHC) surge devido à necessidade de se dispor de um conjunto de informações sistematizadas sobre a incidência de câncer no hospital. Segundo o Instituto Nacional do Câncer.

são fontes sistemáticas de informações, instalados em hospitais gerais ou especializados em oncologia, com o objetivo de coletar dados referentes ao diagnóstico, tratamento e evolução dos casos de neoplasia 
maligna atendidos nessas instituições, sejam públicas, privadas, filantrópicas ou universitárias. (INCA, 2010b, p.17):

Os RHCs no Brasil foram se consolidando como fonte de informações para o planejamento e a assistência ao paciente oncológico, após a publicação da portaria GM/MS n. 3.535 de 1998, BRASIL (2004), do Ministério da Saúde. A portaria n.3535, ao definir critérios para cadastramento dos Centros de Tratamento de Alta Complexidade (CACONs), definiu como obrigatória, a realização do registro de câncer.

O RHC coleta, armazena e analisa dados dos prontuários de todos os pacientes atendidos no hospital, com diagnóstico confirmado de câncer. As informações do RHC são utilizadas para a realização de monografias da Especialização em Cancerologia, para a produção de trabalhos científicos pelo corpo clínico do serviço de oncologia e como base de informação para a pesquisa clínico-epidemiológica institucional. (SOUZA; MIRANDA e SOARES, 2012).

A coleta de dados deve abranger todas as especialidades médicas da instituição. As informações do RHC são coletadas através da anamnese e evolução clínica do paciente. Assim, é de extrema importância que os prontuários sejam completos e fidedignos. Devem conter dados sobre escolaridade, profissão, procedência, naturalidade, cor, tabagismo, etilismo, histórico familiar de câncer, estadiamento TNM 5 (que avalia o grau de disseminação), topografia específica classificada pela Classificação Internacional de Doenças para Oncologia (CID-0), que apresenta a sua localização no corpo humano, etc. (INCA, 2019a).

A qualidade da informação do prontuário do paciente é o fator determinante para a qualidade do RHC, pois o registro é um espelho das informações contidas neste documento. Assim, qualidade significa completude e acurácia de informações nas quais é de extrema importância que o médico relate localização do tumor (CID), tipo histológico (M-), estadiamento clínico/patológico, tratamentos realizados, resultado do tratamento e seguimento do paciente que informa se o paciente está vivo ou se foi a óbito por causa da doença (SOUZA; MIRANDA e SOARES, 2012). D'Alessandro, Antoniazzi e Abreu (2010, p.414), afirmam que "as informações dos registros hospitalares de câncer são de suma importância para o planejamento de ações de prevenção e controle do câncer[...]".

O Registro Hospitalar além de atender ao propósito da administração do hospital, de programas de controle do câncer, tem foco em particular no paciente, ao

\footnotetext{
$5 \mathrm{~T}$ = tumor primário, $\mathrm{N}$ = localizaçção do tumor, $\mathrm{M}$ = presença ou ausência de metástases à distância.
} 
oferecer valioso recurso para acompanhar e avaliar a qualidade do trabalho realizado nos hospitais e os resultados finais alcançados no tratamento do câncer. (INCA, 2010b).

\subsection{ESTIMATIVAS DE CÂNCER NO BRASIL}

O INCA, em sua publicação sobre as estimativas de câncer para o triênio 2020/2022 no Brasil, relatou que se espera a ocorrência de aproximadamente 625 mil casos novos de câncer (450 mil, excluindo os casos de câncer de pele não melanoma), sendo que deste número são esperados um total de 309.230 casos novos para o sexo masculino e 316.140 para o sexo feminino. 0 câncer de maior incidência nas mulheres será o câncer da mama com 66.280 casos novos, com um risco estimado de 61,61 casos novos a cada 100 mil mulheres. Já para os homens estimam-se 65.840 casos novos de câncer da próstata, com um risco estimado de 62,95 casos novos a cada 100 mil homens. (INCA, 2019b).

As informações da figura 1, foram geradas através dos diversos RHCs espalhados pelo Brasil, que coletam os dados dos prontuários de pacientes com câncer e passam esta informação através de um banco de dados: o Sistema Informatizado de Apoio ao RHC (SIS-RHC), a secretaria de saúde que por sua vez, repassa a informação ao INCA.

Figura 1: Distribuição proporcional dos dez tipos de câncer mais incidentes estimados para 2020 por sexo, exceto pele não melanoma*

\begin{tabular}{|c|c|c|c|c|c|c|c|}
\hline Próstata & 65.840 & $29,2 \%$ & & & Mama feminina & 66.280 & $29,7 \%$ \\
\hline Cólon e Reto & 20.540 & $9,1 \%$ & Homens & Mulheres & Cólon e Reto & 20.470 & $9,2 \%$ \\
\hline Traqueia, Brônquio e Pulmão & 17.760 & $7,9 \%$ & & & Colo do útero & 16.710 & $7,5 \%$ \\
\hline Estômago & 13.360 & $5,9 \%$ & & & Traqueia, Brônquio e Pulmão & 12.440 & $5,6 \%$ \\
\hline Cavidade Oral & 11.200 & $5,0 \%$ & & & Glândula Tireoide & 11.950 & $5,4 \%$ \\
\hline Esôfago & 8.690 & $3,9 \%$ & & & Estômago & 7.870 & $3,5 \%$ \\
\hline Bexiga & 7.590 & $3,4 \%$ & & & Ovário & 6.650 & $3,0 \%$ \\
\hline Linfoma não Hodgkin & 6.580 & $2,9 \%$ & & & Corpo do útero & 6.540 & $2,9 \%$ \\
\hline Laringe & 6.470 & $2,9 \%$ & & & Linfoma não Hodgkin & 5.450 & $2,4 \%$ \\
\hline Leucemias & 5.920 & $2,6 \%$ & & & Sistema Nervoso Central & 5.230 & $2,3 \%$ \\
\hline
\end{tabular}

Fonte: INCA (2020b)

A utilização do prontuário, além da assistência ao paciente, visa a realização de estudos epidemiológicos, a vigilância e rastreamento do câncer, serve de fonte de 
diagnóstico e evolução da doença, tratamentos realizados, além disso, é fonte de informações para realização do RHC e RCBP. Assim, os registros de câncer, por meio da coleta de dados em prontuários de pacientes da Oncologia, são importantes para as publicações anuais do INCA sobre as estimativas de câncer no Brasil.

\section{CONSIDERAÇÕES FINAIS}

Por meio do estudo, percebemos a importância de ações para garantir a qualidade, segurança e guarda da informação dos prontuários dos pacientes e estas devem ser contínuas. Uma vez que, esse documento serve de fonte de informação para melhor atenção ao paciente com câncer, em primeiro lugar, mas apresenta também outras finalidades secundárias, ligadas à pesquisa e à promoção de ações de vigilância de saúde. Há grande interesse pelos prontuários dos pacientes, que ao contrário de outros documentos, não decai após a fase corrente. 0 interesse pelas informações do prontuário pode ser aumentado por seus valores secundários, como fonte de informação para pesquisa e planejamento de políticas públicas.

A atividade de coleta de dados, realizado pelo Registro Hospitalar de Câncer (RHC) é essencial para realização de estudos epidemiológicos, na produção científica, no planejamento hospitalar, no fluxo de atendimento de pacientes oncológicos, na criação de políticas, campanhas de prevenção e detecção precoce do câncer em determinada população. O RHC é viabilizado devido às informações dos prontuários, se constituem importante instrumento de apoio a formulação da Política Nacional de Atenção Oncológica, à avaliação da qualidade de atividade assistencial e como subsídio para elaboração de pesquisa clínica.

0 prontuário é documento que contém os dados para a assistência aos pacientes com tumores malignos. Com as informações registradas no prontuário em papel ou em formato eletrônico, é possível realizar análises dos dados, traçar o perfil dos pacientes atendidos no hospital, evidenciar aspectos demográficos, mostrar os recursos que são usados no diagnóstico e tratamento, acompanhar a evolução da doença e o estado geral dos pacientes ao longo do tempo.

O PEP se configura como uma necessidade atual. A tendência é que os registros médicos se firmem no formato eletrônico, desde que sejam vencidas as barreiras que 
assegurem a qualidade dos prontuários eletrônicos como documentos arquivísticos, além da própria política de saúde e infraestrutura hospitalar e corpo técnico.

Concluímos que a organização da informação em saúde, por meio da gestão de arquivos de prontuários, é necessária e fundamental para proporcionar uma melhor assistência ao paciente com câncer. E que a tipologia prontuário do paciente (seja impresso ou eletrônico) deve ter seus valores primários e secundários, melhores considerados, para que o documento de fato cumpra todo o ciclo de vida, atendendo primeiramente à sua instituição criadora, mas também aos estudos da área médica e, estudos para a criação de políticas públicas de saúde.

\section{REFERÊNCIAS}

BARRETO, Eliana Maria Teixeira. Acontecimentos que fizeram a história da oncologia no Brasil: INCA. Revista Brasileira de Cancerologia, Rio de Janeiro, v. 51, n.3, p.267-75, 2005.

BARROWS, Randolph. C.; CLAYTON, Paul. D. Privacy, confidentiality, and electronic medical records. Journal of the American Medical Informatics Association, v.3, n.2, p.139-48, mar./apr. 1996.

BELLOTO, Heloísa Liberalli. Como fazer análise diplomática e análise tipológica de documentos de arquivo. São Paulo: Arquivo do Estado e Imprensa Oficial do Estado, 2002. 114p.

BERTOLLI FILHO, Cláudio. Prontuários médicos: fonte para o estudo da história social da medicina e da enfermidade. História, ciências, saúde-Manguinhos, Rio de Janeiro, v.3, n.1,p.173-80, jun. 1996.

BEZERRA, Selene Maria. Prontuário Eletrônico do Paciente: uma ferramenta para aprimorar a qualidade dos serviços de saúde. Meta: Avaliação, Rio de Janeiro, v. 1, n. 1, p. 73-82, jan./abr. 2009.

BRASIL. Lei no 13.787 , de 27 de dezembro de 2018. Dispõe sobre a digitalização e a utilização de sistemas informatizados para a guarda, o armazenamento e o manuseio de prontuário de paciente. Diário Oficial da União, Brasília, 28. dez. 2018. Disponível em http://www.planalto.gov.br/ccivil_03/_ato2015-2018/2018/lei/L13787.htm.Acesso em: maio 2020.

BRASIL. Ministério da Saúde. Secretaria de Assistência à Saúde. Instituto Nacional de Câncer. Registro hospitalar de câncer: dados dos hospitais do INCA, relatório anual 1994/1998. Rio de Janeiro: INCA; 2004. 
CONSELHO FEDERAL DE MEDICINA (CFM - Brasil). Código de Ética Médica: resolução CFM nº. 1931, de 17 de setembro 2009. Brasília, 2010. Versão de bolso. Disponível em: http://www.portalmedico.org.br/novocodigo/campanha.asp. Acesso em: maio 2020.

CONSELHO FEDERAL DE MEDICINA (CFM - Brasil). Código de Ética Médica: resolução CFM no 1.246 de 08 de janeiro de 1988. Diário Oficial da União, Brasília, 26 jan. 1988. Disponível em:

http://www.portalmedico.org.br/include/codigo_etica/codigo_etica2.asp. Acesso em: maio 2020.

CONSELHO FEDERAL DE MEDICINA (CFM - Brasil). Resolução no 1.638 de 2002. Define prontuário médico e torna obrigatória a criação da Comissão de Revisão de Prontuários nas instituições de saúde. Diário Oficial da União, Brasília. 9 ago. 2002.

CONSELHO FEDERAL DE MEDICINA (CFM - Brasil). Resolução no 1.821 de 2007. Aprova as normas técnicas concernentes à digitalização e uso dos sistemas informatizados para a guarda e manuseio dos documentos dos prontuários dos pacientes, autorizando a eliminação do papel e a troca de informação identificada em saúde. Diário Oficial da União, Brasília, 23 nov. 2007.

CONSELHO FEDERAL DE MEDICINA (CFM - Brasil).Resolução no 1931 de 2009. Aprova o Código de Ética Médica. Diário Oficial da União,Brasília, 24 set 2009.Disponível em : http://www.portalmedico.org.br/resolucoes/cfm/2009/1931_2009.htm. Acesso em: maio 2020.

CONSELHO NACIONAL DE ARQUIVOS(CONARQ). Resolução no 22, de 30 de junho de 2005. Dispõe sobre as diretrizes para a avaliação de documentos em instituições de saúde. Diário Oficial da União, Brasília, n.126, 04 jul. Disponível em :

http://conarq.gov.br/resolucoes-do-conarq/264-resolucao-n-22,-de-30-de-junho-de2005.html. Acesso em: maio 2020.

CONSELHO REGIONAL DE MEDICINA DO DISTRITO FEDERAL. Prontuário médico do paciente: guia para uso prático. Brasília: CRM, 2006. 94p. Disponível em: http://www.saudedireta.com.br/docsupload/1370271458PEP.pdf. Acesso em: maio 2020.

COSTA, Claudio Giulliano Alves da. Cartilha sobre Prontuário Eletrônico: a

Certificação de Sistemas de Registro Eletrônico de Saúde.São Paulo: Conselho Federal de Medicina (CFM) e da Sociedade Brasileira de Informática em Saúde, 2012. Disponível em :http://portal.cfm.org.br/crmdigital/Cartilha_SBIS_CFM_Prontuario_Eletronico_fev_201 2.pdf. Acesso em: 30 maio 2020.

D’ALESSANDRO, Thays Aparecida Leão; ANTONIAZZI, Berenice Navarro; ABREU, Daisy Maria Xavier de. Registros hospitalares de câncer de Minas Gerais: análise de consistência das bases de dados. Cadernos saúde coletiva, Rio de Janeiro. v.18, n.3, p. 410-7, 2010.

DURANTI, Luciana. Registros documentais contemporâneos como provas de ação.

Estudos Históricos. Rio de Janeiro. v.7, n.13, p.49-64, 1994. 
GALLIGIONI, Enzo. et al. Development and daily use of an electronic oncological patient record for the total management of cancer patients: 7 years' experience. Annals of oncology. London. v.20, n.2, p:349-52, 2009.

GIL, Antonio Carlos. Métodos e técnicas de pesquisa social. 4 ed. São Paulo: Atlas, 1994. 207 p.

GREENE, Mark. O poder do significado: a missão arquivística na era pós-moderna. In: Neves, Marta Eloísa Melgaço; Negreiros, Leandro Ribeiro. (Orgs.). Documentos Eletrônicos: fundamentos arquivísticos para a pesquisa em gestão e preservação. Belo Horizonte: Secretaria do Estado da Cultura de Minas Gerais, Arquivo Público Mineiro, 2008. cap.1, p.15-32.

INSTITUTO NACIONAL DE CÂNCER (INCA).Câncer no Brasil: dados dos registro de base populacional, v.4.Rio de Janeiro: INCA, 2010a. 488 p. Disponível em https://www.inca.gov.br/sites/ufu.sti.inca.local/files//media/document//registro_de_b ase_populacional_completo.pdf. Acesso em: 28 maio de 2020.

INSTITUTO NACIONAL DE CÂNCER (INCA).Registros hospitalares de câncer: planejamento e gestão. 2 ed.Rio de Janeiro: INCA, 2010b. 536 p.Disponível em https://www.inca.gov.br/sites/ufu.sti.inca.local/files//media/document//registroshospitalares-de-cancer-2010.pdf. Acesso em: 28 maio de 2020.

INSTITUTO NACIONAL DE CÂNCER (INCA). Estadiamento. Última modificação: 22/04/2019a .Disponível em : https://www.inca.gov.br/estadiamento. Acesso em: 21 maio de 2020.

INSTITUTO NACIONAL DE CÂNCER (INCA). Vigilância de Câncer. Última modificação: 21/09/2018 |09h55. Disponível em : https://www.inca.gov.br/numeros-decancer/vigilancia-de-cancer. Acesso em: 30 de maio 2020.

INSTITUTO NACIONAL DE CÂNCER. (INCA). Estimativa 2020: incidência de câncer no Brasil. Rio de Janeiro : INCA, 2019b. 120 p. Disponível em : https://www.inca.gov.br/sites/ufu.sti.inca.local/files/media/document/estimativa2020-incidencia-de-cancer-no-brasil.pdf. Acesso em: 21 maio de 2020.

KANAS Gena, et al. Use of electronic medical records in oncology outcomes research. Clinicoecon Outcomes Res. v.2, p.1-14. 2010.

MASSAD, Eduardo; MARIN, Heimar de Fatima; AZEVEDO NETO, Raymundo Soares de. (Ed.). 0 prontuário eletrônico do paciente na assistência, informação e conhecimento médico. São Paulo, Março de 2003.213p. Disponível em : http://www.sbis.org.br/biblioteca_virtual/prontuario.pdf. Acesso em: maio 2020.

MUELLER, Suzana Pinheiro Machado. A ciência, o sistema de comunicação científica e a literatura científica. In: CAMPELLO, Bernadete Santos; CENDÓN, Beatriz Valadares; KREMER, Jeannette Marguerite. (Orgs.). Fontes de informação para pesquisadores e profissionais. Belo Horizonte: UFMG, 2000, p. 21-34. 
ROSSEAU, Jean-Yves, COUTURE, Carol. Os fundamentos da disciplina arquivística. Lisboa: Publicações Dom Quixote, 1998.

SHORTLIFFE, Edward H.; BARNETT, G. Octo. Biomedical Data: Their Acquisition, Storage, and Use. In: SHORTLIFFE, Edward H.; CIMINO, James J. (Ed). Biomedical Informatics: Computer Applications in Health Care and Biomedicine. 4th ed. London: Springer-Verlag, 2014. cap.2, p.39-66.

SILVA, Marcelo Lúcio; VIRGINIO JUNIOR, Luiz Aparecido. (Eds.) Manual de Certificação para Sistemas de Registro Eletrônico em Saúde: Versão 4.2, certificação 2016. São Paulo: SBIS, 2016.Disponívewl em :

http://www.sbis.org.br/certificacao/Manual_Certificacao_SBIS-CFM_2016_v4-2.pdf. Acesso em: 28 maio de 2020.

SOUZA, Amanda Damasceno de; MIRANDA, Márcia Borges; SOARES, Aleida Nazareth. 0 Registro Hospitalar de Câncer (RHC) do Serviço de Oncologia da Santa Casa: uma análise dos dados consolidados. In: SIMPÓSIO MINEIRO DE ONCOLOGIA, 14, 2012. Belo Horizonte. Anais... São Paulo: Âmbito Hospitalar, 2012. Pôster no9.

TEIXEIRA, Josenir. Notas jurídicas sobre o prontuário do paciente. São Paulo: GT,2007.168p.

\section{Agradecimentos}

Agradecemos a Márcia Borges de Miranda, Bióloga responsável pelo Registro Hospitalar de Câncer da Santa Casa de Misericórdia de Belo Horizonte, pelas contribuições valorosas na realização da pesquisa e na correção do artigo. 